SCIENTIFIC LETTER

\title{
Prospective longitudinal assessment of late anthracycline cardiotoxicity after childhood cancer: the role of diastolic function
}

\author{
I Dorup, G Levitt, I Sullivan, K Sorensen
}

Heart 2004;90:1214-1216. doi: 10.1136/hrt.2003.027516

$\Lambda$ nthracyclines are highly effective in the treatment of childhood malignancies, but are potentially cardiotoxic at all dose ranges. Early studies suggested that as many as $65 \%$ of acute lymphoblastic leukaemia (ALL) survivors have subclinical abnormalities of left ventricular (LV) systolic function at medium term follow up. More recent reports have shown that the prevalence of cardiac abnormalities is much lower with cumulative anthracycline doses of less than 250$300 \mathrm{mg} / \mathrm{m}^{2}{ }^{1-4}$

It has been suggested that diastolic changes precede systolic dysfunction in various conditions and predict later deterioration. Similarly, LV restriction has been suggested to be a late functional event after anthracycline exposure. Most reports of anthracycline cardiotoxicity are cross-sectional studies of heterogeneous diagnostic groups receiving various anthracycline schedules. ${ }^{5}$ We obtained prospective longitudinal diastolic data in two well defined cohorts of long term survivors of ALL and Wilms' tumor (WT) and compared this to systolic function. Both cohorts underwent echocardiographic evaluation in 1991-92 and again in 1995-96.

\section{METHODS}

The original cohort consisted of 120 ALL survivors, comprising a convenience sample of 40 survivors from each of three treatment groups receiving a total dose of 90,180 or $270 \mathrm{mg} / \mathrm{m}^{2}$ of daunorubicin. Initially, 106 of these subjects underwent investigation of diastolic filling on average 6.2 years after anthracyline treatment, and 94 patients underwent follow up leaving comparative diastolic data available in 88 patients.

From 110 WT patients treated between 1970 and 1990, 90 underwent investigation of diastolic filling in 1991-92, and 78 were reinvestigated in 1995-96, on average 6.7 years and 11.1 years after treatment, respectively. Of these, 66 subjects had complete comparative diastolic data sets. Mean cumulative doxorubicin dose at first study was $303(79) \mathrm{mg} / \mathrm{m}^{2}$.

One hundred healthy children and adolescents were studied on a single occasion using identical echocardiographic methods.

LV diastolic performance was assessed from pulsed wave mitral Doppler flow, allowing measurements of peak early velocity (E), peak atrial velocity (A), E/A ratio (EA), and E deceleration time (EDEC). Isovolumetric relaxation time (IVRT) was measured as the time interval between the first high frequency component of the second heart sound (on the phonocardiogram) and the onset of early ventricular filling. The same two observers, blinded to the type of malignancy and anthracycline dose, performed the examinations and analysis.

Cardiac measurements from the control subjects provided a normal range within which the measurements from the patients could be expressed in standard deviation units (z scores)—for example, IVRTz. Thus the mean z score for all such parameters for the group of normal controls is 0 with an $\mathrm{SD}$ of 1 . All $\mathrm{z}$ scores were corrected for the regression with age. The results were expressed as mean (SD) and for statistical analyses the two sample $t$ tests and univariate and multiple regression techniques were used.

\section{RESULTS}

Serial data for each diastolic parameter are shown in fig 1. In the ALL group, E, EA, IVRT, and EDEC increased significantly between studies. Similar, but more pronounced, changes were observed in the WT patients. Univariate regression between anthracycline dose and diastolic parameters showed that patients who had received higher anthracycline doses had reduced $\mathrm{E}$ at the first study $(r=-0.22, \mathrm{p}<0.005)$, and reduced E $(r=-0.22, \mathrm{p}<0.005)$ and EA $(r=-0.16$, $\mathrm{p}<0.05)$ at the second study. At the second assessment, IVRT prolonged with increasing dose $(r=0.24, \mathrm{p}<0.005)$, and anthracycline doses above $250 \mathrm{mg} / \mathrm{m}^{2}$ were likely to be associated with prolonged IVRT (IVRTz above 0).

Multivariate regression identified dose $(r=0.26$, $\mathrm{p}<0.001)$ and age at treatment $(r=0.17, \mathrm{p}=0.03)$ as independent predictors of IVRT at second study. Dose also correlated with EA $(r=-0.19, \mathrm{p}=0.02)$. Diagnosis, sex, and follow up interval were not associated with any diastolic measures. None of the potential risk factors were predictors of a difference between the two studies.

In view of these findings, and our observation that systolic function seems to deteriorate with cumulative doses above $250 \mathrm{mg} / \mathrm{m}^{2}$, the present cohort was divided into two subgroups according to cumulative doses below and above $250 \mathrm{mg} / \mathrm{m}^{2}$. Patients receiving higher doses had significantly lower E velocity and EA as well as longer IVRT at second study.

To test the hypothesis that diastolic abnormalities precede or predict systolic abnormalities or progressive systolic dysfunction, temporal changes in fractional shortening were correlated with initial diastolic parameters. Patients with fractional shortening $(<25 \%)$ at second study had significantly lower E at the first study, compared to those with normal fractional shortening $(\geqslant 30 \%)$, otherwise all other diastolic indices were not significantly different. Multivariate analysis showed that determinants for fractional shortening at the second study were only dose $(r=-0.41, \mathrm{p}<0.001)$ and $\mathrm{E}$ at first study $(r=0.21, \mathrm{p}=0.005)$. None of the patients with normal E velocity at the first study had reduced fractional shortening at the second study, but only 13\% of those with a subnormal (defined as fractional shortening less

Abbreviations: A, peak atrial velocity; ALL, acute lymphoblastic leukaemia; $E$, peak early filling velocity; EDEC, E wave deceleration time; IVRT, isovolumetric relaxation time; LV, left ventricular; WT, Wilms' tumour. 

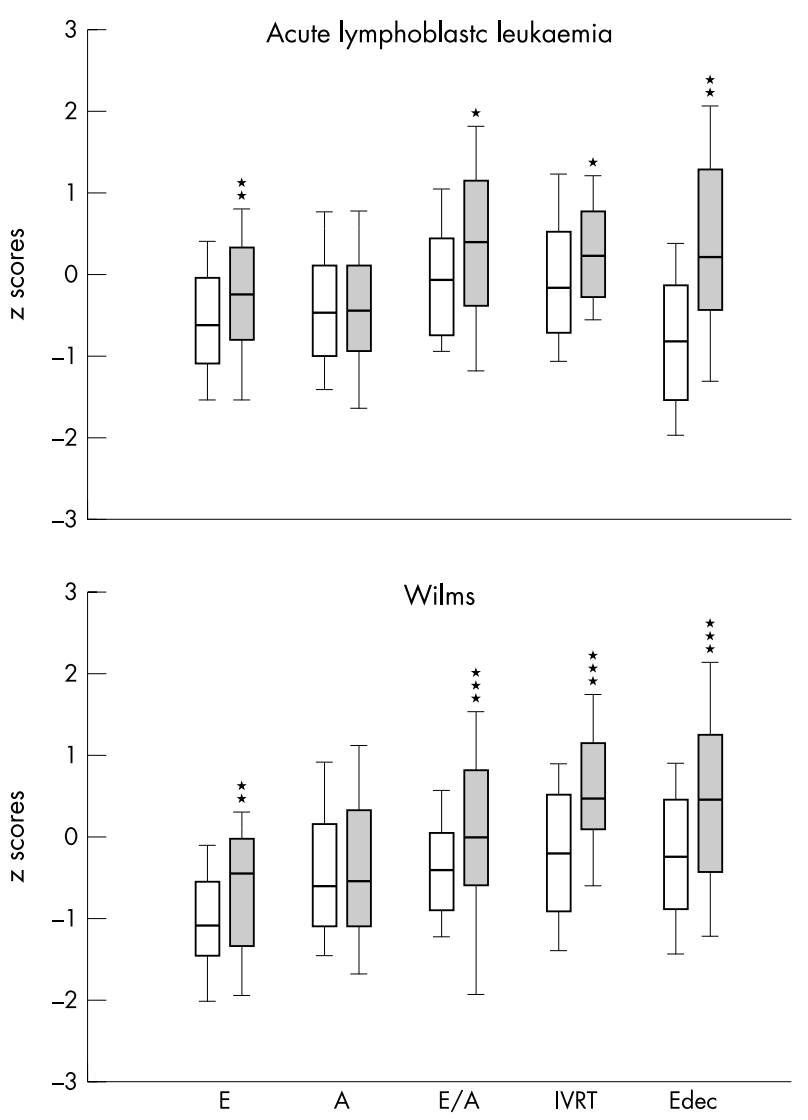

Figure 1 Box plot of transmitral flow variables for $88 \mathrm{ALL}$ patients (upper panel) and 66 WT patients (lower panel) studied on two occasions. The first study is represented by open bars and the second study by filled bars. The horizontal lines of the plot mark the 10th, 25 th, 50th, 75th and 90th centiles, and the box encompasses the 25th through 75th centiles. Variables are expressed in standard deviation units $(z)$. ${ }^{*} \mathrm{p}<0.05 ;{ }^{* *} \mathrm{p}<0.005 ;{ }^{* * *} \mathrm{p}<0.001$; paired $t$ test.

than mean fractional shortening -2 SDs of the controls) E velocity at the first study had reduced fractional shortening at the second study.

\section{DISCUSSION}

Alterations in Doppler derived diastolic filling characteristics in anthracycline treated children have been reported previously. Some have described frequent abnormalities, ${ }^{5}$ with numerically small changes, directionally variable and not confined to those receiving anthracyclines. Others have not been able to detect any differences from controls, ${ }^{2}$ neither at rest nor during dobutamine stress testing. All but one of these studies of diastolic function in anthracycline treated children has included a variety of different diagnostic groups receiving various anthracycline schedules. Rammeloo and colleagues $^{2}$ studied children with ALL only, but found no indices of diastolic dysfunction. However, anthracycline doses were low $\left(100 \mathrm{mg} / \mathrm{mg}^{2}\right)$.

Restrictive LV filling has been proposed as a consequence of chronic progressive myocardial anthracycline induced damage, but our observations in patients treated with moderate anthracycline doses and a follow up interval of 10-11 years, would question this hypothesis, at least in the dose range and follow up interval studied. In WT patients, the observed prolongation in EDEC and IVRT between studies was significant compared to healthy controls. This filling pattern or change in filling pattern is difficult to define, but possibly indicates a directional shift towards impaired relaxation (although E and EA increased) rather than a change towards a restrictive filling pattern.

The present data showed a relation between anthracycline dose and reduced E, prolonged IVRT, and prolongation of IVRT between studies. This might suggest that anthracycline dose is a risk factor for the development of impaired $\mathrm{LV}$ relaxation. Increased dose is a risk factor for systolic abnormalities. ${ }^{134}$ The observation that cumulative doses under $250 \mathrm{mg} / \mathrm{m}^{2}$ may not be associated with deterioration in diastolic function supports our own observations on systolic performance in the same cohorts $^{4}$ and other reports. $^{2}$

Our data offer a unique opportunity to test whether diastolic abnormalities correlate with or predict subsequent changes in systolic function. Multivariate analysis of determinants of fractional shortening showed that only dose and E velocity were predictors for reduced fractional shortening at follow up. However, the presence of abnormal E velocity at first study had low sensitivity for predicting abnormal systolic function, although normal E velocity was a highly specific determinant.

Current knowledge regarding anthracycline cardiotoxicity is primarily based on systolic indices. Interpretation of systolic data is relatively straightforward as functional indices all change unidirectionally with worsening systolic function. In contrast, diastolic dysfunction cannot be described by one index only, but depends on an interaction between a number of measures which do not change in a linear way but rather in different directions. Assessment of pulmonary venous flow patterns would theoretically have made our data more comprehensive and perhaps made analysis easier. This measure was, however, not obtained when these cohorts were first studied. Tissue Doppler imaging may prove advantageous when trying to evaluate cardiac function more completely.

Our findings confirm that changes in diastolic indices are seen in survivors of childhood cancer treated with anthracyclines. Correlation between increasing anthracycline dose and diastolic abnormalities suggesting impaired relaxation was evident, and reduced E velocity correlated weakly with subsequently impaired systolic function. None of the diastolic indices, however, had any useful predictive value for impaired systolic function. Based on our experience with prospective systolic and diastolic analysis in the same cohorts, it is our current opinion that routine assessment of diastolic flow characteristics does not offer useful extra information.

\section{Authors' affiliations}

I Dorup, K Sorensen, Department of Cardiology, Aarhus University Hospital (SKS), Aarhus N, Denmark

G Levitt, Department of Haematology/Oncology, Great Ormond Street Hospital for Children NHS Trust, London, UK

I Sullivan, Department of Cardiology, Great Ormond Street Hospital for Children NHS Trust

Correspondence to: Dr Inge Dorup, Department of Cardiology B, Aarhus University Hospital (SKS), Brendstrupgaardsvej, DK-8200 Aarhus N, Denmark; inge.dorup@dadlnet.dk

Accepted 10 December 2003

\section{REFERENCES}

1 Sorensen K, Levitt G, Sebag-Montefiore D, et al. Cardiac function in Wilms' tumor survivors. J Clin Oncol 1995;13:1546-56.

2 Rammeloo LAJ, Postma A, Sobotka-Plojhar MA. Low-dose daunorubicin in induction treatment of childhood acute lymphoblastic leukemia: no long-term 
cardiac damage in a randomised study of the Dutch childhood leukemia study group. Med Ped Oncol 2000;35:136-9.

3 Sorensen K, Levitt G, Bull C, et al. Anthracycline dose in childhood acute lymphoblastic leukaemia: issues of early survival versus late cardiotoxicity.

J Clin Oncol 1997;15:61-8.
4 Sorensen K, Levitt GA, Bull C, et al. Late anthraracycline cardiotoxicity after childhood cancer. A prospective longitudinal study. Cancer 2003;97:1991-8. 5 Bu'Lock FA, Mott MG, Oakhill A, et al. Left ventricular diastolic filling patterns associated with progressive anthracycline-induced myocardial damage: a prospective study. Pediatr Cardiol 1999;20:252-63.

\section{IMAGES IN CARDIOLOGY}

Intravascular ultrasound features of the "crush" technique for treatment of bifurcational coronary artery stenoses

A 60 year old man presented for elective treatment of a bifurcation lesion, involving both the mid left anterior descending artery (LAD) and the ostium of a large diagonal branch (Dl) (panel B). Intravascular ultrasound examination confirmed the narrowing of Dl, suggesting that stenting the LAD alone would result in significant side branch impairment (panels A and C).

Using an 8 French guide catheter, after preinflation, two paclitaxel drug eluting stents (Taxus, Boston Scientific) were advanced to the LAD and Dl. The Dl stent was then inflated while the LAD stent was left in position. Then, after removing the Dl balloon and guidewire, the LAD stent was deployed, "crushing" the proximal part of the Dl stent behind it.

After rewiring Dl, a "kissing balloon" postdilatation was undertaken.

The final IVUS run confirmed an excellent angiographic appearance (panel E), with the Dl ostium wide open (panel D), and visualised the "crushed" proximal portion of the Dl stent (three layers of stent struts in the LAD proximal to the bifurcation) (panel F).
The procedure was quick and uneventful, with no enzyme rise, and the patient was discharged home the next day. Clopidogrel treatment was recommended for at least six months.

Dealing with bifurcations remains one of the challenges for interventional cardiology. In the past, randomised comparisons between strategies involving stenting only the main branch (with provisional stenting of the side branch) and various approaches using two stents, have consistently shown the inferiority of the latter, due to very high rates of restenosis, in spite of better acute angiographic results. In the era of drug eluting stents, however, encouraging preliminary data have been reported for the "crush" technique, involving sequential inflation of two stents.

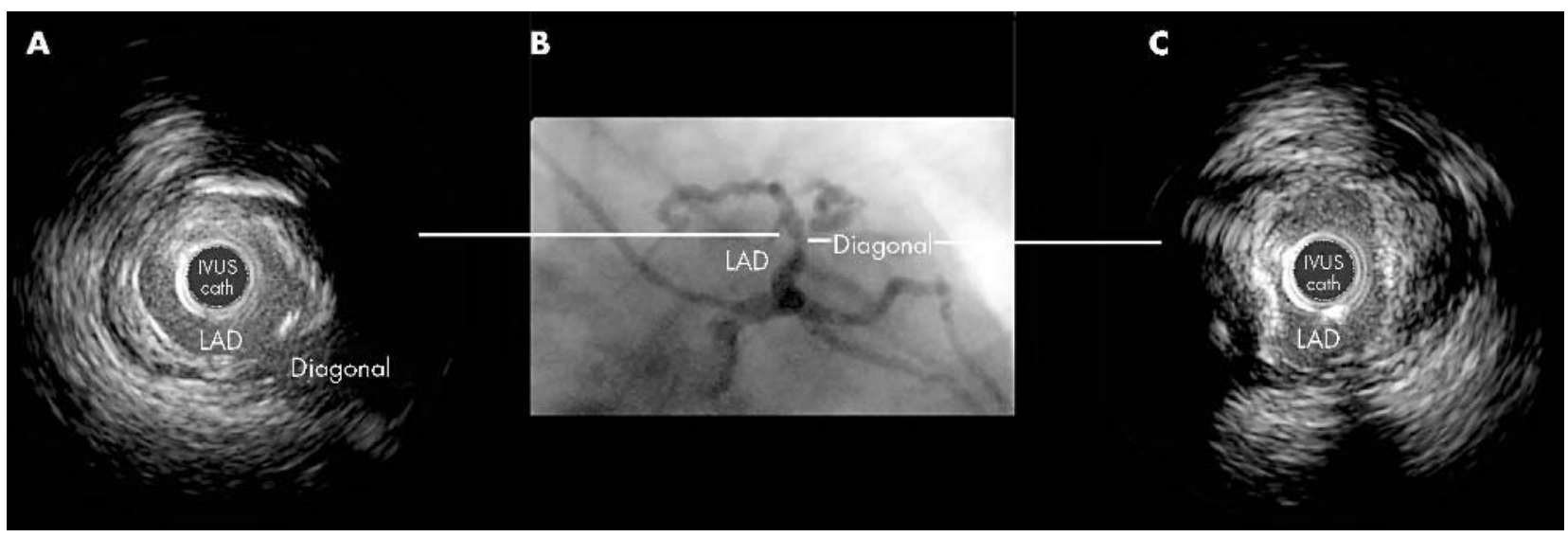

I Porto

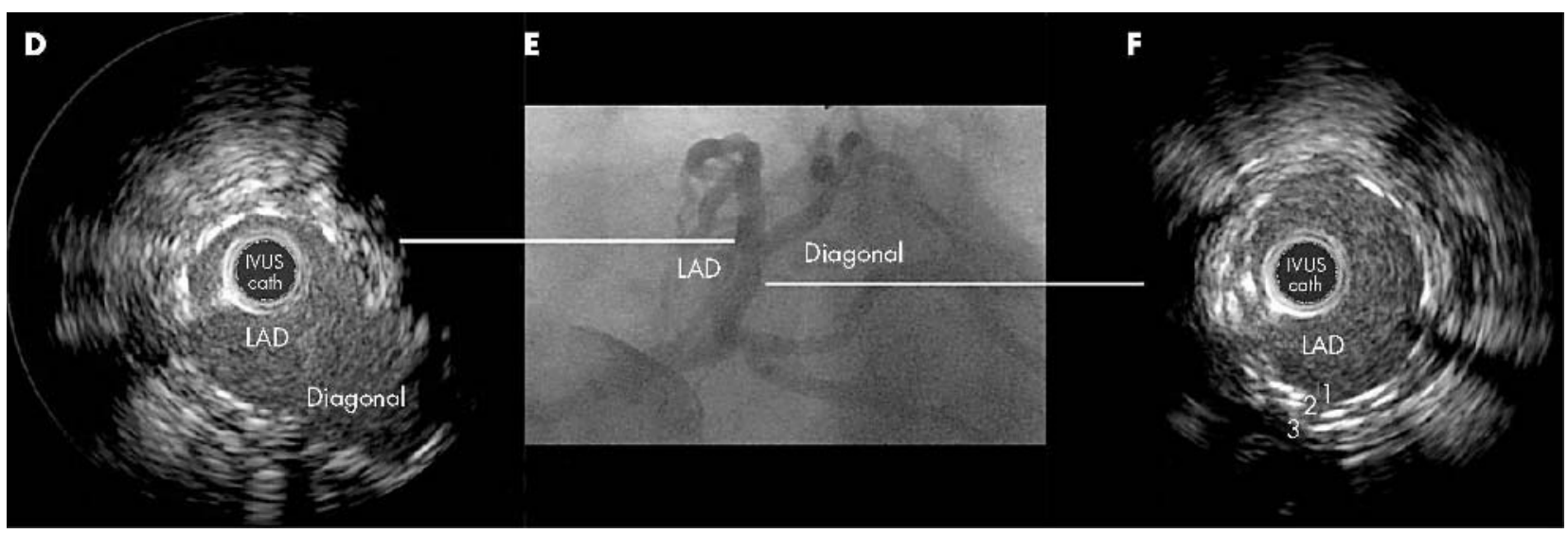

